\title{
Dps promotes survival of nontypeable Haemophilus influenzae in biofilm communities in vitro and resistance to clearance in vivo
}

\author{
Bing Pang ${ }^{1}$, Wenzhou Hong ${ }^{1 \dagger}$, Nancy D. Kock ${ }^{2,3}$ and W. Edward Swords ${ }^{1 *}$ \\ ${ }^{1}$ Department of Microbiology and Immunology, Wake Forest University Health Sciences, Winston-Salem, NC, USA \\ ${ }^{2}$ Department of Comparative Medicine, Wake Forest University Health Sciences, Winston-Salem, NC, USA \\ ${ }^{3}$ Department of Pathology, Wake Forest University Health Sciences, Winston-Salem, NC, USA
}

\author{
Edited by: \\ Daoguo Zhou, Purdue University, \\ USA \\ Reviewed by: \\ Margaret E. Bauer, Indiana \\ University School of Medicine, USA \\ Jason W. Johnston, University of \\ Kentucky, USA \\ *Correspondence: \\ W. Edward Swords, Department of \\ Microbiology and Immunology, \\ Wake Forest University Health \\ Sciences, 5303 Hanes Biomedical \\ Research Building, Medical Center \\ Boulevard, Winston-Salem, \\ NC 27157, USA. \\ e-mail:wswords@wfubmc.edu \\ ${ }^{\dagger}$ Present address: \\ Department of Otolaryngology, \\ Medical College of Wisconsin, \\ Milwaukee, WI, USA
}

Nontypeable Haemophilus influenzae (NTHi) is a common airway commensal and opportunistic pathogen that persists within surface-attached biofilm communities. In this study, we tested the hypothesis that bacterial stress-responses are activated within biofilms. Transcripts for several factors associated with bacterial resistance to environmental stress were increased in biofilm cultures as compared to planktonic cultures. Among these, a homolog of the DNA-binding protein from starved cells (dps) was chosen for further study. An isogenic NTHi 86-028NP dps mutant was generated and tested for resistance to environmental stress, revealing a significant survival defects in high-iron conditions, which was mediated by oxidative stress and was restored by genetic complementation. As expected, NTHi 86-028NP dps had a general stress-response defect, exhibiting decreased resistance to many types of environmental stress. While no differences were observed in density and structure of NTHi 86-028NP and NTHi 86-028NP dps biofilms, bacterial survival was decreased in NTHi 86-028NP dps biofilms as compared to the parental strain. The role of dps persistence in vivo was tested in animal infection studies. NTHi 86-028NP dps had decreased resistance to clearance after pulmonary infection of elastase-treated mice as compared to NTHi 86-028NP, whereas minimal differences were observed in clearance from mock-treated mice. Similarly, lower numbers of NTHi 86-028NP dps were recovered from middle-ear effusions and bullar homogenates in the chinchilla model for otitis media (OM). Therefore, we conclude that Dps promotes bacterial survival within NTHi biofilm communities both in vitro and in chronic infections in vivo.

Keywords: Haemophilus influenzae, Dps, stress, biofilm, COPD, otitis media

\section{INTRODUCTION}

Nontypeable Haemophilus influenzae (NTHi) is a nearly universal nasopharyngeal commensal that can cause opportunistic airway infections, including bronchopulmonary infections in patients with chronic obstructive pulmonary disease (COPD) and otitis media $(\mathrm{OM})$ and sinusitis infections in children. In the United States, epidemiologic data indicate that COPD affects over 10 million adults, and is a major cause of death, hospitalizations, and emergency-room visits (Wedzicha and Seemungal, 2007). Patients with COPD frequently experience opportunistic airway infections that are a major complicating factor in the clinical course and management of the disease (Sethi and Murphy, 2001), and much recent data have correlated bacterial and viral infections with the inflammatory exacerbations that are the hallmark of this disease (Sethi et al., 2002, 2007; Watanabe et al., 2004; Chin et al., 2005; Berenson et al., 2006a,b; Kweon et al., 2006; Moghaddam et al., 2007; Tumkaya et al., 2007). OM is among the most common pediatric infections worldwide, affecting the majority of all children at least once by 3 years of age
(Klein, 2000). Clinical presentations of OM include chronic and recurrent infections that feature multiple incidents of overt disease over the course of months to years (Paradise et al., 1997). The bacteria causing COPD-related and OM infections are opportunists that are normally found within the nasopharyngeal microbiota, including nontypeable Haemophilus influenzae, Moraxella catarrhalis, and Streptococcus pneumoniae (Sethi and Murphy, 2001; Murphy et al., 2004, 2005a; Pichichero, 2005; Pichichero and Casey, 2007).

Persistent infections caused by NTHi and other mucosal opportunists involve the formation of biofilm communities that enhance bacterial resistance to clearance (Swords et al., 2004; Hong et al., 2007a,b, 2009). Biofilms are typically defined as surface-adherent communities that confer resistance to environmental stress and/or clearance mechanisms (Hall-Stoodley and Stoodley, 2009). In the case of NTHi, it is also clear that specific bacterial factors are important in the formation and maturation of biofilms both in vitro and within animal models (Swords et al., 2004; Jurcisek et al., 2005, 2007; Starner et al., 2006; Hong et al., 
2007a,b, 2009; Jurcisek and Bakaletz, 2007; Armbruster et al., 2009, 2010, 2011). For example, NTHi biofilms contain variants expressing specific subsets of lipooligosaccharide (LOS) glycoforms that are essential to biofilm formation and/or maturation (Greiner et al., 2004; Swords et al., 2004; Hong et al., 2007a,b). Additional factors associated with NTHi biofilms include pili (Murphy and Kirkham, 2002; Jurcisek et al., 2007), extracellular DNA (Jurcisek and Bakaletz, 2007), and, possibly, a non-LOS extracellular polysaccharide (Greiner et al., 2004; Jurcisek et al., 2005). NTHi biofilms have been observed in tissue from patients with chronic OM (Hall-Stoodley et al., 2006) and rhinosinusitis (Sanderson et al., 2006), and in the case of OM a chronic infection involving biofilms has been recapitulated in animal models (Post, 2001). Within the context of COPD, the strongest indication of NTHi biofilms has been provided by expression of biofilmassociated proteins such as peroxiredoxin in sputa from patients with COPD (Murphy et al., 2005b). In recent work, we have also demonstrated that multicellular NTHi communities are present within the lungs of elastase-treated mice, from which clearance is significantly impaired (Pang et al., 2008a).

In this study, we used a microarray approach to identify transcripts with increased levels in NTHi 86-028NP biofilms as compared to planktonic growth. One of the factors with increased expression was a homolog of the DNA-binding protein associated with starvation (Dps). An NTHi 86-028NP dps null mutant was generated, and shown to have increased susceptibility to environmental stress, consistent with prior work with other bacterial species. This mutant was also compared with the parental strain using in vitro biofilm assays and in animal infection models for COPD-related infections and OM. The results clearly show that Dps promotes survival of NTHi $86-028 \mathrm{NP}$ within biofilm communities, as well as resistance to host clearance in vivo. These data indicate that the NTHi stress-response may promote survival during chronic infection, and may thus be an important target for antimicrobial therapy during chronic infections.

\section{MATERIALS AND METHODS BACTERIAL CULTURE AND MANIPULATION}

The bacteria, primers, and plasmids used in this study are presented in Table 1. NTHi $86-028 \mathrm{NP}$ is a well-defined clinical isolate for which a complete genomic sequence, and baseline data for biofilm formation and infection models are available (Bakaletz et al., 1988; Harrison et al., 2005). NTHi bacteria were cultured in brain-heart infusion media (Difco) or minimal medium, supplemented with hemin $(0.01 \mathrm{~g} / \mathrm{ml}, \mathrm{ICN}$ Biochemicals $)$ and nicotinamide adenine dinucleotide $(0.01 \mathrm{~g} / \mathrm{ml}$, Sigma $)$.

\section{MICROARRAY STUDIES}

To identify transcripts increased during biofilm growth, NTHi 86-028NP was cultured in a commercial continuous-flow biofilm system (Stovall) for varying times as indicated in the text. Planktonic cultures from the same inocula were cultured in the same preparation of supplemented $\mathrm{BHI}$, and harvested during mid-logarithmic and stationary-phase. Total RNA was prepared from NTHi using the RNeasy kit (Qiagen), quantified using a NanoDrop spectrophotometer, and analyzed by gel electrophoresis. RNA from six independently derived samples was used to probe a commercial total genomic NTHi 86-028NP microarray (Nimblegen). The full results of the microarray studies are presented as Appendix information. Results were confirmed using quantitative real-time RT-PCR as indicated in the text.

\section{CLONING OF $d p s$ (NTHi1817) AND GENERATION OF NTHi $d p s$ MUTANT}

Genomic DNA was purified from NTHi 86-028NP using a commercial kit (Qiagen). A $5 \mathrm{~kb}$ amplicon containing the $d p s$ open reading frame was amplified using the PCR and primers p-dpsF and p-dpsR (Table 1), then cloned into pCR2.1 (Invitrogen) to generate plasmid pCR-dps, which was then confirmed by nucleotide sequencing. A second amplification was performed using primers p-dpskoF and p-dpskoR, such that the majority of the $d p s$ open reading frame was deleted and a blunt-ended cloning

Table 1 | Primers, plasmids, and bacterial strains used in this study.

\begin{tabular}{|c|c|c|}
\hline Designation & Description & Reference \\
\hline p-dpsF & 5'-CTATCAAGCACCGAAAAGAGC-3' & This study \\
\hline p-dpskoF & 5'-GGTAGCCCCGCACATTAGTA-3' & This study \\
\hline p-dpskoR & 5'-TTGCAGTAAGTGAGGCACAAG-3' & This study \\
\hline p-IRAF & 5'-GTGCCGATCAACGTCTCATTTTC-3' & Jones et al., 2002 \\
\hline \multicolumn{3}{|l|}{ PLASMIDS } \\
\hline pCR-dps & NTHI 1817 (dps) cloned into pCR2.1 vector & This study \\
\hline pdps-1 & dps::kan null allele & This study \\
\hline pIRAdps-1 & NTHI 1817 (dps) cloned into pIRA-gm vector & This study \\
\hline pIRA-gfp & hel-gfp cloned into pIRA-cm vector & Hong et al., 2007a \\
\hline
\end{tabular}


site was generated. A kanamycin-resistance cassette was then

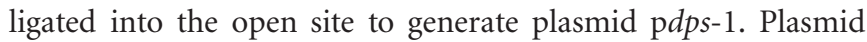
$\mathrm{p} d p s-1$ was linearized by digestion with NotI and introduced into NTHi $86-028 \mathrm{NP}$ by a colony transformation method that we have described previously (Hong et al., 2007a,b). Transformants were selected by growth on supplemented $\mathrm{BHI}$ agar containing $5 \%$ Fildes' reagent (Difco) and $0.5-1.5 \mu \mathrm{g} / \mathrm{ml}$ chloramphenicol, and confirmed by PCR and sequence analysis. To complement the $d p s$ mutation, the insert from $p d p s-1$ containing the entire $d p s$ open reading frame was purified by EcoRI digestion fragment and cloned into the pIRA-gm vector containing an intergenic, noncoding region of $H$. influenzae genomic DNA (Jones et al., 2002) to generate pIRAdps-1, and confirmed by restriction mapping and nucleotide sequence analysis. This construct was linearized and introduced into NTHi $86-028 \mathrm{NP} d p s$ by transformation as above, with selection on supplemented BHI agar containing $2 \mu \mathrm{g} / \mathrm{ml}$ gentamicin and 5\% Fildes' reagent. Transformants were confirmed by PCR of the IRA intergenic region as described previously (Jones et al., 2002), and phenotypic complementation was confirmed as described in the text.

\section{STRESS-RESPONSE EXPERIMENTS}

Resistance of NTHi 86-028NP, NTHi 86-028NP dps, and the complemented NTHi $86-028 \mathrm{NP} d p s$ pIRA- $d p s$ strain to oxidative, osmotic, temperature, and iron stress was performed essentially as described previously for similar studies with other bacterial species (Halsey et al., 2004; Nair and Finkel, 2004). For oxidative stress, the strains were cultured in the presence of $\mathrm{H}_{2} \mathrm{O}_{2}$ as indicated in the text. Temperature stress was tested by incubation in differing temperatures as indicated in the text. Iron stress was tested using $\mathrm{FeSO}_{4}$ with and without added pyridone. After each treatment, bacterial viability was assessed by plate-count.

\section{BIOFILM ASSAYS}

To assess role(s) of $d p s$ in biofilm structural parameters, NTHi 86-028NP and NTHi 86-028NP $d p s$ were transformed with pIRA- $g f p$ as described previously for constitutive expression of green fluorescent protein. Both strains were cultured in Stovall continuous-flow chambers and visualized by confocal microscopy at $12,24,48$, and $72 \mathrm{~h}$ time-points. Biofilm structural measures were obtained using COMSTAT software, as described previously and analyzed by one-way analysis of variance (ANOVA) with a post-hoc test of significance. No significant differences in any measures of biofilm structure were observed (data not shown). To assess bacterial viability within biofilms, biofilms were cultured as above and stained with BacLight Live-Dead ${ }^{\mathrm{TM}}$ reagent (Molecular Probes) at 24, 48, and $72 \mathrm{~h}$, essentially as described (Allen et al., 2005; Jurcisek and Bakaletz, 2007).

\section{INFECTION STUDIES}

To test the role of $d p s$ in the resistance of NTHi 86-028NP to pulmonary clearance, an elastase-treatment mouse model for COPD/emphysema was used (Pang et al., 2008a,b). C57/BL6 mice (Jackson Laboratories) were anesthetized with Avertin (2,2,2tribromoethanol) and treated with a $50 \mu \mathrm{l}$ pulmonary bolus of elastase (3 U, Sigma) delivered via an intratracheal route, as we have recently described (Pang et al., 2008a). Control mice received vehicle (PBS). Our recent work shows that this treatment elicits pulmonary damage and fibrosis that is pathologically similar to COPD/emphysema (Pang et al., 2008a). Two weeks following treatment, the mice were anesthetized with Avertin and intratracheally infected with $\sim 10^{7} \mathrm{CFU}$ of NTHi $86-028 \mathrm{NP}$ or NTHi 86-028NP dps. Groups of mice (5/group) were euthanized at $24 \mathrm{~h}$ or $48 \mathrm{~h}$ post-infection, and the lungs removed. One lung was homogenized, serially diluted, and plated onto supplemented $\mathrm{BHI}$ agar containing $3 \mu \mathrm{g} / \mathrm{ml}$ vancomycin for plate-count. Platecount data were analyzed by unpaired $t$-test analysis with Welch's correction for unequal variance; groups with $P$ values $\leq 0.05$ were deemed significantly different from control. The other lungs were fixed in 4\% paraformaldehyde/PBS for histopathology and cryosection. The elastase-treatment and infection protocols were approved by the Wake Forest University Health Sciences Animal Care and Use committee.

OM infections were carried out using the chinchilla model, essentially as described previously (Hong et al., 2007a,b, 2009). Healthy animals were obtained from Rauscher's chinchilla ranch (Larue, $\mathrm{OH}$ ), and acclimated to the WFUHS vivarium for $7 \mathrm{~d}$ to minimize stress related to shipping. Animals were anesthetized by isofluorane inhalation and inoculated with $\sim 10^{3}$ CFU of NTHi 86-028NP or NTHi $86-028 \mathrm{NP} d p s$ via transbullar injection. All chinchilla infection protocols were approved by the WFUHS Animal Care and Use Committee. All animals were monitored by otoscopic examination throughout the course of the infection studies. At designated time-points post-infection animals were euthanized, and the superior bullae were excised to expose the middle-ear chamber. Effusion fluids were collected when present, and the bullae were excised and homogenized as indicated in the text. Bacterial load in effusions and associated with tissues were determined by plate-count. Portions of biofilm material were excised and stained using the Live-Dead ${ }^{\mathrm{TM}}$ reagent (Molecular Probes) according to the manufacturers' instructions, and essentially as described previously (Jurcisek and Bakaletz, 2007; Hong et al., 2009; Juneau et al., 2011).

\section{HISTOPATHOLOGY}

Portions of fixed murine lung tissue were dehydrated and embedded in paraffin according to standard methods. Sections $(5 \mu \mathrm{m})$ were cut from paraffin-embedded blocks with a microtome and mounted from warm water $\left(40^{\circ} \mathrm{C}\right)$ onto adhesive microscope slides. After serial incubation with xylene to remove paraffin followed by rehydration, tissue sections were stained with hematoxylin and eosin for histopathologic assessment. Stained slides were provided as a blinded set to a veterinary pathologist (N.K.), and were scored for markers of inflammation (neutrophilic influx, edema, epithelial damage, and necrosis).

\section{RESULTS}

\section{TRANSCRIPTOME PROFILING OF NTHi 86-028NP BIOFILMS}

Transcript levels in NTHi 86-028NP biofilms cultured for varying lengths of time in continuous media flow were compared with logarithmic-phase and stationary-phase planktonic controls using a total-genome NTHi 86-028NP microarray (Nimblegen). The results, presented in Table 2, indicated that expression of approximately $1.5 \%$ of the open reading frames 
Table 2 | Transcripts with increased expression in NTHi 86-028NP biofilm.

\begin{tabular}{llll}
\hline ORF & Identification & Microarray & Real-time RT-PCR \\
\hline NTHI0022 & Conserved hypothetical acid-induced glycyl radical enzyme & 4.3 & 5.9 \\
NTHI0270 & Probable formate transporter & 3.2 & 3.2 \\
NTHI0481 & Periplasmic chelated iron-binding protein & 2.3 & ND \\
NTHI0668 & Co-chaperonin GroES & 4.6 & 0.9 \\
NTHI0669 & Chaperonin GroEL & 2.9 & ND \\
NTHI0705 & Peroxiredoxin/glutaredoxin & 2.2 & ND \\
NTHI1012 & Thiol:disulfide interchange protein DsbA & 2.4 & 5.1 \\
NTHI1028 & ClpB & 2.7 & 2.4 \\
NTHI1214 & Conserved putative d-carboxymuconolactone decarboxylase subunit & 2.1 & ND \\
NTHI1230 & Cytochrome c nitrate reductase & 2.4 & ND \\
NTHI1588 & ImpA & 2.1 & ND \\
NTHI1929 & Chaperone protein DnaK & 4.6 & 2.8 \\
NTHI1817 & DNA-binding ferritin-like protein (Dps) & 4.8 & 5.9 \\
\hline
\end{tabular}

Values represent fold change relative to stationary-phase planktonic cultures.

in the genome were differentially expressed during biofilm as opposed to planktonic growth (for full data see Appendix information). In particular, 25 transcripts associated with bacterial stress-response and anaerobic metabolism were increased during biofilm growth. These results indicate that oxidative stress is increased in biofilm cultures, as peroxiredoxin and $d p s$ transcript levels were increased. Additional groups of genes with increased transcript levels included those associated with anaerobic/hypoxic conditions, including several genes associated with formate uptake and metabolism. Quantitative real-time RT-PCR on independently cultured biofilm and control cultures indicated a similar level of increase for this transcript as observed in the microarray experiments. For this study, we focused on the role of $d p s$.

\section{HOMOLOGY OF NTHi 86-028NP dps}

NTHI1817 was compared with $d p s$ open reading frames from other bacterial sequences in GenBank using the BLAST algorithm (Figure 1). The predicted amino acid sequence from this open reading frame had significant homology to $d p s$ sequences from other species, with a high degree of conservation in the predicted DNA binding regions (Figure 1A). Comparison with sequenced $H$. influenzae genomes revealed nearly $100 \%$ homology among different strains (Figure 1B). Thus, the NTHI1817 dps gene appears to be highly conserved.

\section{MUTATION OF dps DECREASES RESISTANCE TO OXIDATIVE STRESS}

In other bacterial species, mutation of $d p s$ confers susceptibility to a variety of environmental stresses that include oxidative stress (Halsey et al., 2004). Therefore, an isogenic dps mutant was generated in the NTHi 86-028NP strain background and compared with the parental strain for resistance to hydrogen peroxide according to standard methodology. The results clearly show increased killing of NTHi 86-028NP $d p s$ by $\mathrm{H}_{2} \mathrm{O}_{2}$ as compared with the parental strain (Figure 2A). Of note, genetic complementation of $d p s$ significantly restored oxidative stress-resistance (Figure 2B).
NTHi $86-028 \mathrm{NP}$ dps also exhibited a significant survival defect in media containing excess iron as compared with the parental strain (Figure 3A). These results are consistent with increased hydroxyl radical generated via reduction of iron in the Fenton reaction. To define the mechanism for enhanced oxidative killing of NTHi 86-028NP $d p s$, we performed stress-resistance experiments in the presence of the iron chelator $2^{\prime}, 2$-dyridyl $(1 \mathrm{mM})$ or the free-radical scavenger dimethyl sulfoxide (DMSO) (Vattanaviboon and Mongkolsuk, 1998; Halsey et al., 2004). Both treatments rescued the oxidant sensitive phenotype of NTHi 86$028 \mathrm{NP} d p s$ (Figure 3). Thus, the oxidant susceptibility of NTHi $86-028 \mathrm{NP} d p s$ is conferred by increased hydroxyl radical, most likely resulting from iron oxidation.

Additional experiments showed that NTHi 86-028NP $d p s$ had a general stress-response defect, including increased susceptibility to osmotic, temperature, and $\mathrm{pH}$ stress (data not shown). These results are consistent with prior work in other bacterial species.

\section{Dps PROMOTES NTHi SURVIVAL WITHIN BIOFILMS in vitro}

As the microarray data indicated increased expression of $d p s$ in NTHi 86-028NP biofilms, the impact of $d p s$ mutation on biofilm formation and bacterial viability was assessed. While the quantity of adherent biomass was increased in biofilms formed by NTHi 86-028NP $d p s$ as compared with the parental strain (Figure 4A), there was a significant decrease in viable bacteria within the biofilm for NTHi $86-028 \mathrm{NP}$ dps (Figure 4B). Consistent with this result, a greater proportion of non-viable bacteria were observed in biofilms formed in continuous-flow chamber slide apparatus by NTHi $86-028 \mathrm{NP} d p s$ as compared with the parent (Figures 4C,D). Based on these results, we conclude that $d p s$ promotes NTHi survival within biofilm.

\section{Dps PROMOTES NTHi PERSISTENCE in vivo}

In order to define the contribution of $d p s$ to persistent infection with NTHi, animal infection studies were performed using the elastase-treated mouse model for COPD/emphysema and the chinchilla model for OM. Bacterial counts were significantly 
A

B

HI 86-028NP HIRdKW20 HIR 2866

HI 22 . 1-21 HI 3655 HIPittAA HIPitteE HIPittII HIPittGG HIPittHH

HI 86-028NP HIRdKW 20 HIR 2866 HI 22. 1-21 HI 3655 HIPittAA HIPittEE HIPittII HIPittGG HIPitthH

HI 86-028NP HIRdKW 20 HIR2866 HI 22 . 1-21 HI 3655 HIPittAA HIPittEE HIPittII HIPittGG HIPittHH
DNA-binding region I - - - - MSKTSIGIDKVQSAELADKLNELIATYOVFYTNVRGYHWNIKGVNFF 47 --MAMSNIGLDKEIAKKLADELNGLIATYOVFYTNVRGYHWNVRGVNFF 47 ----------MTTT IGLDRNSSKQLATELNNLLASYQI FYMNVRGYHWNIKGVNFF 46 -------------MVNS IGLDKVSAEKLAKELNGLLASYQVFYTNVRGYHWNIKGVSFF 46 -------MKT LNYTHLEEKGANT IVLSLQQLLADFQ I HYANLRGF HWNIKGHGFF 48 ---------MKKILEVTGLKEQQVAPVVKGLSGLLADLQVYYSNLRGFHWNIRGAEFF 49

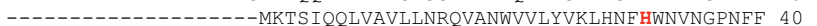
-_-_-_-_-_-_-_--MKT INSVDTKEF LNHQVANLNVFTVKI HOI HWYMRGHNF F 40 --------------MSATTTLKEQVLTTLKREQANAVVMYLNYKKYHWLTYGPLFR 42 MSTAKLVKSKATNLLYTRNDVSDSEKKATVELLNRQVIQFIDLSLITKQAHWNMRGANFI 60 MKTHK----------TKNDLPSNAKSTVIGI LNESLASVIDLALVTKQAHWNLKGPQFI 49 MTSFT-----------IPGLSDKKASDVADLLQKQLSTYNDLHLTLKHVHWNVVGPNFI 48 DNA-binding region II

$\overline{A L H A K F E}$ TYTNLVARVDEVAERIITIGYTPNNAYSOYLKISRIKED--IAVSEAOECLS 105 ALHAKFEEIYTNLVARVDEVAERILTLGYTPNNAYSQYLKISRIKED--IAVSEAQECLS 105 ELHAKFEEVYTDLVEKVDGVAERILTLGYTPNNAFSQYLTLARIKED--IAVSNAITCLK 105
ELHAKFEEIYDDLVTKVDEIAERILTLGHTPONAFSOYLTKSOIOEH--IGVSAAOECLR 105 ELHAKFEEIYDDLVVKVDEIAERILTLGYTPSNAFSEYLTQSQIQEH--INVTNAQDCLN 104 ELHAKFEEIYDDLVVKVDEIAERILTLGYTPDNAFSQYLQVSLVKED--IGASSAQHCLT 104 VLHSKFEDLYNGAAEKVDEIAERILMLGGT PANKYSDYLKMAQIKEV--DGVNKADDALN 106 VLHEQYEKMYDDLAGK IDEVAERILQLGGKPENRFSEYLKVAEVKEE--HELVCAASTLK 107 TLHEKFEELYTEASGHIDTLAERVISIGGSPIATLAASLEEASIKEA--TGGESAAEMVS 98 SLHVKFEELYNEASQYVDELAERILAVGGNPVGTLTECLEQS IVKEA--AKGYSAEQMVE 97 TLHEKMDDLYSEFGEQMDEVAERLLAIGGSPFSTLKEFLENASVEEAPYTKPKTMDQLME 100 DLHLLFEEQGSEVFAMIDELAERSLMLDGQPVADPADYLKVATVTPS--SGQLTVKQMIE 100 AVHEMLDGFRTALIDHLDTMAERAVQLGGVALGTTQVINSKTPLKSYP-LDIHNVQDHLK 119 AVHELLDTFRTQLDNHGDTIAERVVQLGGTALGSLQAVSSTTKLKAYP-TDIYKIHDHLD 108 $\frac{\text { GVHEMIDPQVELVRGY ADEVAERIATLGKSPKGTPGAIIKDRTWDDYS-VERDTVQAHLA }}{:^{\star}:} 107$

GTLQGLKTLLDQQREILAFANNANDEGTASQMSDYIKEQEKLVWMFQ--AACQTCHN--- 160 GTLEGFKVLLAQQREI LALAGEAGDEGTVSQMSDY IKEQEKLVWMFQ--AACQACAS--- 160 GTLNGFKTLLGQQREILALANDANDEGTASQMSDYIKEQEKLVWMFT --AACAECSDYAQ 163 GTLQGFKTLLKQQRS ILAIASDANDEGTASQMS DY I KEHEKLIWMFS--AACESCHA--- 159 GTLEGFQTLLKQQREILALANAAEDEGTASQMSDYIKEQEKLVWMFSSAATCGVCQ---- 160 H ILETYGHLIAEERKILSLASSHNDEVTVAMMS DYLKEQEKMVWMLT ---AYNG------ 157 NVTDTLOI IMAKERAIAEVAGEAGDEVTVDLMIGFLSGQFKLVWMLS---AYATK----- 159 SVVNDFVDLVGELKVARDVADEADDEATADMLDAIEAGLEKHVWMLEAFLE-------- 149 ELSQDFTNISKQLENAIEIAGNAGDDVSEDMF IGMQTSVDKHNWMFKSYLSLEHHHHHH - 156 DLVGTLELLRDEYQQGIELTDKEGDNVTNDML IAFKAS I DKH IWMF KAFLGKAPLE---- 156 EAIANHEL I ITEMHQDAEI ATEAGD IGTADLYTRLVQTHQKHRWFLKEFLAKGDGLVS -- 158 ELADRYAIVANDVRKAIG---EAKDDDTADILTAASRDLDKFLWFIESNIE--------- 167 ALIERYGEVANMIRKAIDDSDEAGDPTTADIFTAASRDLDKSLWFLEA HVQEKS------ 162 ALDLVYNGVIEDTRKSIEK-LEDLDLVSQDLLIAHAGELEKFQWFVRAHLESAGGQLTHE 166

$$
:{ }^{*} \text { : . }
$$

MSKTSIGLDKVQSAELADKLNELLATYQVFYTNVRGYHWNIKGVNFFALHAKFEEIYTNL 60 MSKTSIGLDKVQSAELADKLNELLATYQVFYTNVRGYHWNIKGVNFFALHAKFEEIYTNL 60 MSKTSIGLDKVQSAELADKLNELLATYQVFYTNVRGYHWNIKGVNFFALHAKFEEIYTNL 60 MSKTSIGLDKVQSAELADKLNELLATYQVFYTNVRGYHWNIKGVNFFALHAKFEEIYTNL 60 MSKTS I GLDKVQSAELADKLNELLATYQVFYTNVRGYHWNIKGVNFFALHAKFEEIYTNL 60 MSKTSIGLDKVOSAELADKLNELLATYOVFYTNVRGYHWNIKGVNFFALHAKFEEIYTNL 60 MSKTS IGLDKVQSAELADKLNELLATYQVFYTNVRGYHWNIKGVNFFALHAKFEEIYTNL 60 MSKTSIGLDKVQSAELADKLNELLATYQVFYTNVRGYHWNIKGVNFFALHAKFEEIYTNL 60 MSKTS IGLDKVQSAELADKLNELLATYQVFYTNVRGYHWNIKGVNFFALHAKFEEIYTNL 60 MSKTSIGLDKVQSAELADKLNELLATYQVFYTNVRGYHWNIKGVNFFALHAKFEEIYTNL 60

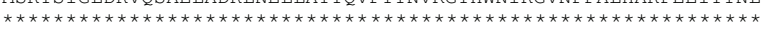

VARVDEVAERILTLGYTPNNAYSQYLKISRIKEDIAVSEAQECLSGTLQGLKTLLDQQRE 120 VARVDEVAERILTLGYT PNNAYSOYLKISRIKEDIAVSEAOECLSGTLQGLKTLIDQQRE 120 VARVDEVAERILTLGYTPNNAYSQYLKISRIKEDIAVSEAQECLSGTLQGLKTLLDQQRE 120 VARVDEVAERILTLGYTPNNAYSQYLKISRIKEDIAVSEAQECLSGTLQGLKTLLDQQRE 120 VARVDEVAERILTLGYTPNNAYSQYLKISRIKEDIAVSEAQECLSGTLQGLKTLIDQQRE 120 VARVDEVAERILTLGYTPNNAYSQYLKISRIKEDIAVSEAQECLSGTLQGLKTLLDQQRE 120 VARVDEVAERILTLGYTPNNAYSQYLKISRIKEDIAVSEAQECLSGTLQGLKTLLDQORE 120 VARVDEVAERIITIGYTPNNAYSOYLKISRIKEDIAVSEAOECISGTLOGLKTLIDQORE 120 VARVDFVAERILTLGYTPNNAYSQYLKISRIKEDIAVSEAQECLSGTLQGLKTLIDQQR VARVDEVAERILTLGYTPNNAYSQYLKISRIKEDIAVSEAQECLSGTLQGLKALIDQQRE 120

ILAFANNANDEGTASQMSDYIKEQEKLVWMFQAACQTCHN 160 I LAFANNANDEGTASQMSDY IKEQEKLVWMFQAACQTCHN 160 ILAFANNANDEGTASOMSDYIKEOEKLVWMFOAACOTCHN 160 ILAFANNANDEGTASOMSDYTKEOEKLVWMFOAACOTCHN 160 ILAFANNANDEGTASOMSDYIKEQEKLVWMFOAACOTCHN 160 $\begin{array}{lll}\text { ILAFANNANDEGTASQMSDYIKEQEKLVWMFQAACQTCHN } & 160 \\ \text { ILAFANNANDEGTASOMSDYIKEOEKLVWMFOAACOTCHN } & 160\end{array}$ $\begin{array}{ll}\text { ILAFANNANDEGTASQMSDY IKEQEKLVWMFQAACQTCHN } & 160 \\ \text { ILAFANNANDEGTASQMSDYIKEQEKLVWMFQAACQTCHN } & 160\end{array}$ $\begin{array}{ll}\text { ILAFANNANDEGTASQMSDYIKEQEKLVWMFQAACQTCHN } & 160 \\ \text { ILAFANNANDEGTASQMSDYIKEQEKLVWMFQAACQTCHN } & 160\end{array}$ ILSFANNANDEGTASQMSDYIKEQEKLVWMFQAACQTCHN 160 ILSFANNANDEGTASQMSDYIKEQEKLVWMFQAACQTCHN 160

FIGURE 1 | BLAST comparison of NTHI1817. (A) Comparison with dps orthologs from other bacterial species. (B) dps is highly conserved among sequenced H. influenzae strains. 


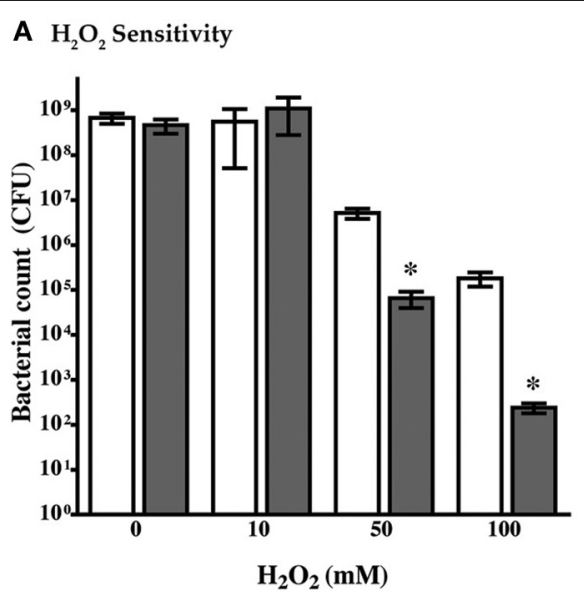

FIGURE 2 | Mutation of $d p s$ sensitizes NTHi 86-028NP $d p s$ to hydrogen peroxide. (A) Comparison of resistance of NTHi 86-028NP (white bars) and NTHi 86-028NP dps (shaded) to hydrogen peroxide. Both strains were suspended to equivalent density $\left(10^{8} \mathrm{CFU} / \mathrm{ml}\right)$ in PBS and treated with the indicated concentration of $\mathrm{H}_{2} \mathrm{O}_{2}$ for 30 min, after which survival was measured by plate-count. Values are means of six independent replicates:
B Genetic complementation

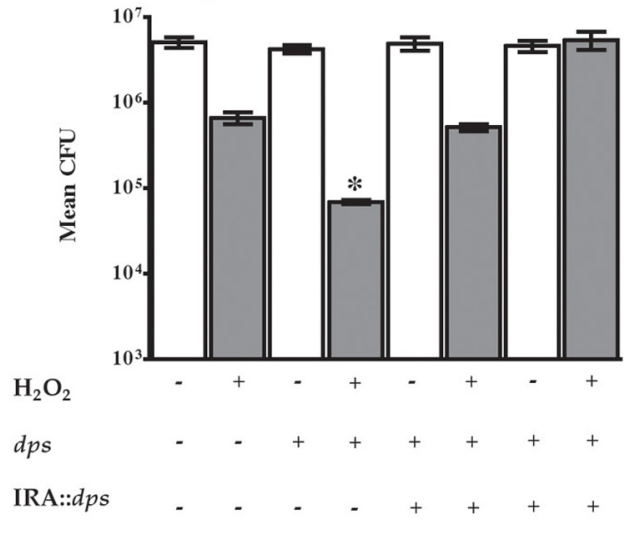

statistical significance was determined by analysis of variance and post-hoc analysis using Bonferroni test. (B) Genetic complementation of dps mutation. An intact copy of the dps open reading frame was introduced into NTHi 86-028NP (white bars) or NTHi 86-028NP dps (shaded) as indicated, and sensitivity to $\mathrm{H}_{2} \mathrm{O}_{2}$ was tested as above. Statistical analysis was performed as in panel (A) above.

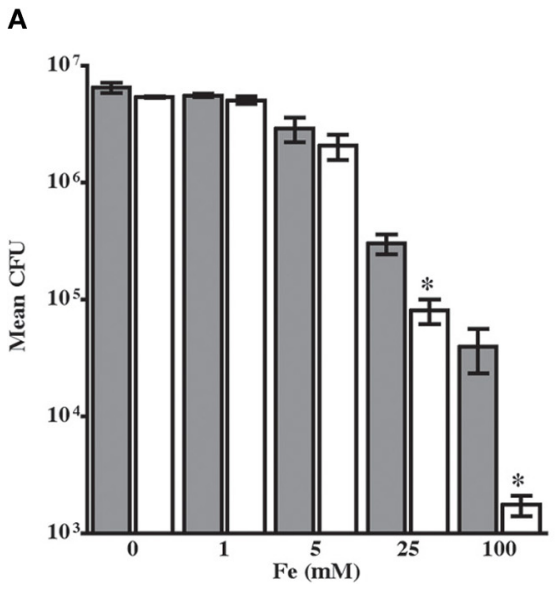

FIGURE 3 | Sensitization of NTHi 86-028NP $d p$ s to hydrogen peroxide occurs via iron-mediated hydroxyl radical production. (A) Growth of NTHi 86-028NP (shaded bars) and NTHi 86-028NP dps (white bars) in varying concentrations of iron. Values are means of six independent replicates; statistical significance was determined by analysis of

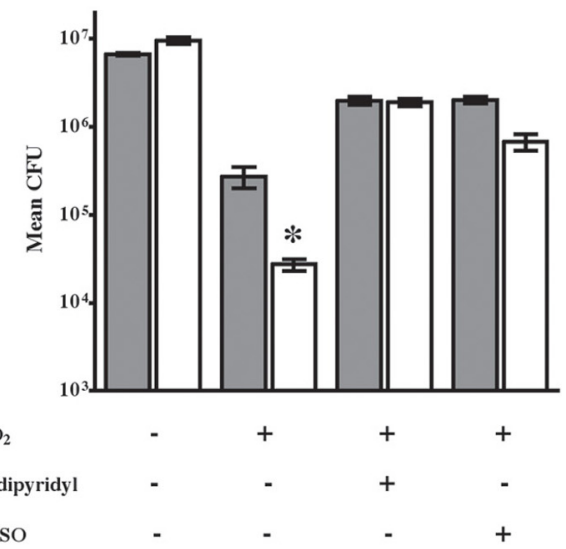

variance and post-hoc analysis using Bonferroni test. (B) Dipiridyl restores resistance of NTHi 86-028NP dps. Values are means of six independent replicates; statistical significance was determined by analysis of variance and post-hoc analysis using Bonferroni test. decreased in elastase-treated mice infected with NTHi 86-028NP $d p s$ as compared with the parental strain (Figure 5A). Notably, the counts obtained from the mice infected with the mutant strain were not significantly greater than those obtained from mock-treated mice receiving vehicle (PBS) instead of elastase. No differences were observed between the infection groups in mock-treated mice. These results are consistent with our previous evaluation of biofilm-deficient NTHi mutant strains in this model system (Pang et al., 2008a). Histopathologic assessment of lung tissues from the infected animals revealed a slight increase in overall markers of inflammation in the mice infected with NTHi 86-028NP $d p s$ as compared with the parental strain (Figures 5B,C).

A comparable persistence defect was observed in the chinchilla infection model (Figure 6). Significantly lower counts were observed in middle-ear effusion fluids (Figure 6A) or middle-ear bullar homogenates (Figure 6B) in animals infected with NTHi 86-028NP $d p s$ as compared with the parental strain, at the 2 week time point post-infection. As the counts obtained at $7 \mathrm{~d}$ postinfection showed no such difference, these results do not appear to be related to initial stages of colonization/infection. Based on these data, we conclude that $d p s$ promotes NTHi survival within biofilms in vivo. 


\section{A Static biofilm formation}

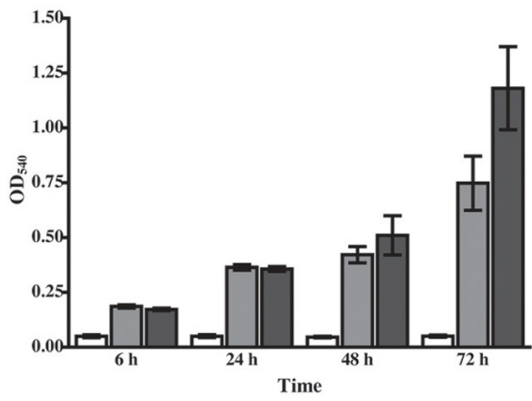

C Live-Dead Stain, NTHi 86-028NP

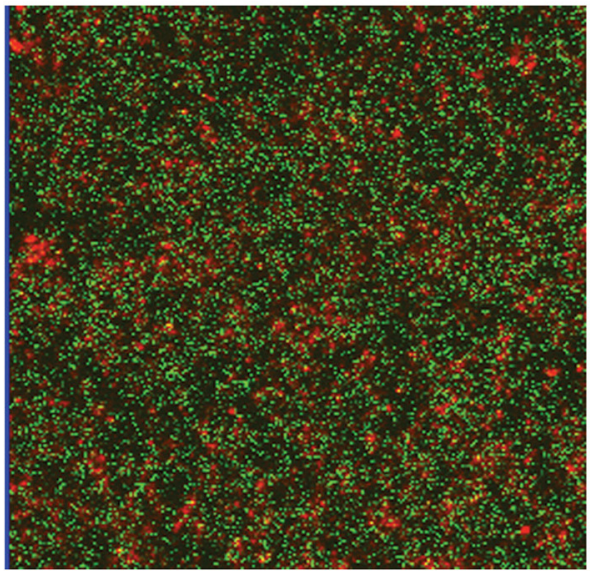

FIGURE 4 | Effect of $d p s$ on NTHi survival in biofilm. (A) Biofilm density. NTHi 86-028NP (gray bars) and NTHi 86-028NP dps (dark bars) were compared using a static biofilm initiation assay (Swords et al., 2004). Values represent absorbance of solubilized biofilm after staining with crystal violet. Control wells with no bacteria are shown as white bars. (B) Bacterial survival within static biofilm. Viability of NTHi 86-028NP (white bars) and NTHi

\section{B Bacterial viability in static biofilm}

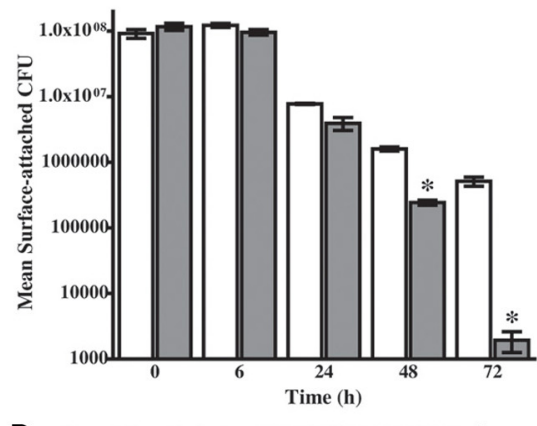

D Live-Dead stain, NTHi 86-028 NP $d p s$

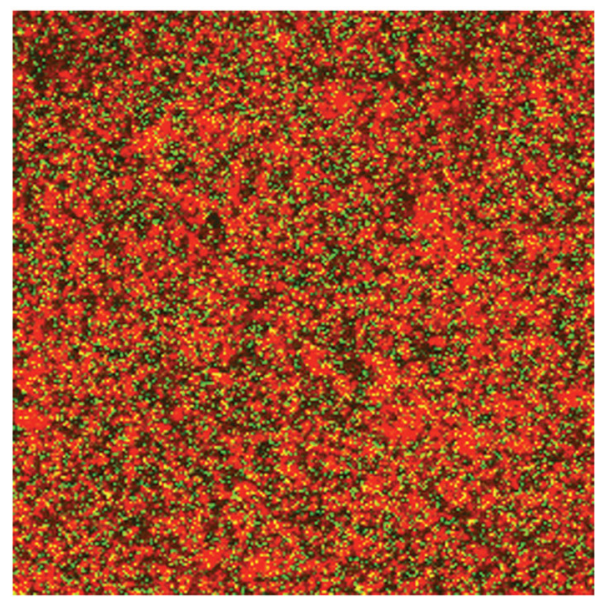

86-028NP dps (shaded bars) was assessed by plate-count of biofilm attached to plastic surface. Values are means of four independent replicates; statistical significance was determined by analysis of variance and post-hoc analysis using Bonferroni test. (C) Viability staining of NTHi 86-028NP biofilm in continuous-flow system $24 \mathrm{~h}$ post-inoculation. (D) Viability staining of NTHi 86-028NP dps biofilm in continuous-flow system $24 \mathrm{~h}$ post-inoculation.

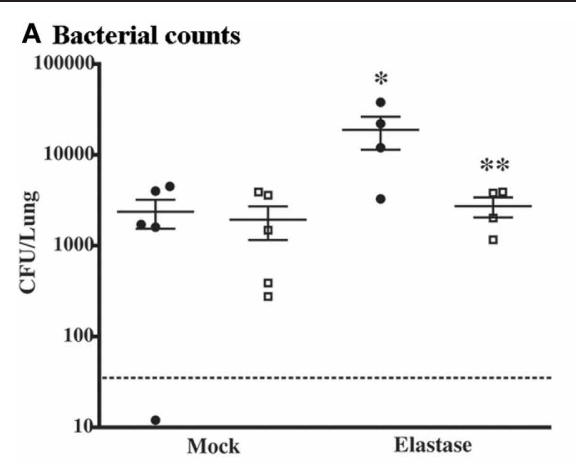

FIGURE 5 | Effect of $\boldsymbol{d p s}$ on NTHi resistance to pulmonary clearance.

(A) Bacterial counts from lungs of elastase-treated mice (Pang et al., 2008a). Animals were pretreated with elastase as described previously and allowed to recover for $14 \mathrm{~d}$ prior to infection. Mock-treated animals received vehicle (PBS). Points represent counts derived from individual mice. Error bars represent standard error of the mean. Statistical significance was determined by nonparametric T-test using Welch's correction.

\section{B Histopathology}

Mock

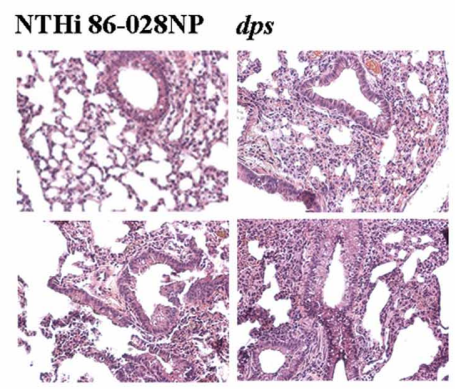

C Histopathology scores

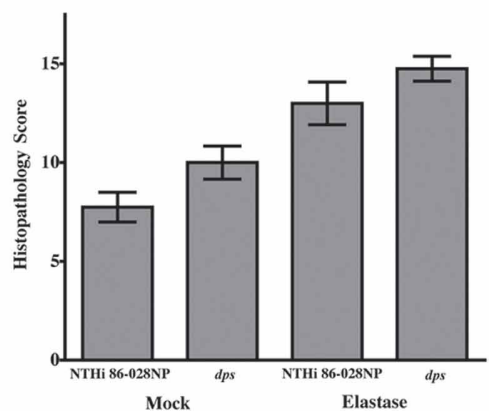

(B) Histopathologic sections from infected lung tissue. Sections were prepared as described previously (Pang et al., 2008a,b) and stained with hematoxylin/eosin according to standard methodology. (C) Inflammatory scores for sections from infected mice. All sections were graded as a blinded set for markers of inflammation as described previously (Pang et al., 2008a,b). Statistical significance was determined by $t$-test as above. 


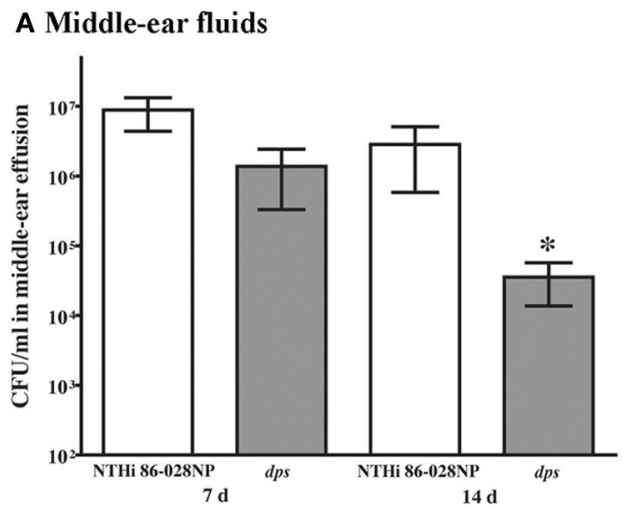

FIGURE 6 | Effect of $\boldsymbol{d p s}$ on NTHi persistence in the chinchilla model for otitis media. Animals were anesthetized and infected with $\sim 1000 \mathrm{CFU}$ as indicated via transbullar injection, and groups (3/group) were euthanized

\section{B Bullar homogenates}

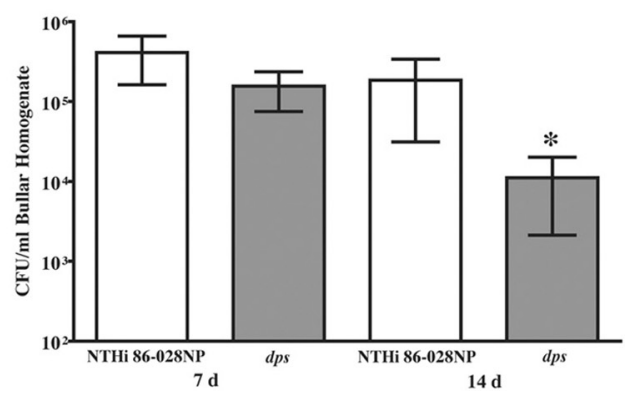

at $7 \mathrm{~d}$ or $14 \mathrm{~d}$ post-infection. Bacterial counts were obtained from middle-ear effusion fluids (A) or bullar homogenates (B). Statistical significance was determined by nonparametric T-test using Welch's correction.

\section{DISCUSSION}

Increased resistance to environmental stress and host clearance are hallmarks of biofilm communities. However, in many cases the mechanism(s) behind this stress resistance is unclear and is probably conferred by multiple means. For example, it is known that biofilms can resist killing by some microbicidal factors due to decreased permeation of the biofilm due to the inherent diffusion barrier provided by the biofilm matrix (Anderl et al., 2000). Additional evidence supports the conclusion that anaerobicity, nutrient limitation, or other environmental conditions within biofilms lead to metabolic resistance of bacteria within a biofilm (Lewis, 2001; Anderl et al., 2003; Kim et al., 2009). Prior work from our laboratory and others in the field has demonstrated roles for a number of different components of the biofilm matrix in resistance of NTHi biofilms to host clearance (Greiner et al., 2004; Swords et al., 2004; Jurcisek et al., 2005, 2007; Hong et al., 2007a,b; Jurcisek and Bakaletz, 2007). In this study, transcript profiling of NTHi biofilms revealed a significant increase in a number of factors involved in bacterial stress-responses, which could conceivably impact the resistance of NTHi biofilms to antibiotics and host clearance. Our data clearly support the conclusion that increased expression of $d p s$ within NTHi biofilms promotes bacterial survival within biofilms and resistance to host clearance. Notably, similar persistence defects were observed for NTHi 86$028 \mathrm{NP} d p s$ in two different animal models for opportunistic NTHi infection. However, it is also important to recognize that the infection results do not indicate a severe defect associated with loss of $d p s$ function. Our interpretation of these results is that $d p s$ is a component of a multifactorial stress-response that occurs in NTHi biofilms, and therefore loss of this one factor, while significant, is not completely deleterious in vivo.

Taken together with our prior work, the results of this study indicate that the resistance phenotype of NTHi biofilms involves both structural and metabolic changes in the bacteria residing within the biofilm community (Swords et al., 2004; WestBarnette et al., 2006; Hong et al., 2007a,b, 2009; Pang et al., 2008a,b; Armbruster et al., 2009). There is considerable evidence for formation of NTHi biofilms during chronic infections that include chronic and recurrent OM (Post, 2001; Hall-Stoodley et al., 2006), rhinosinusitis (Sanderson et al., 2006), and, possibly, chronic bronchitis (Murphy et al., 2005b; Pang et al., 2008a). Understanding the metabolic state of persistent NTHi bacteria within biofilms will be an important objective in learning to better treat chronic NTHi disease. As chronic NTHi infections are among the most common and costly public health problems worldwide, there is a pressing need for additional information regarding the means for NTHi persistence within biofilms.

\section{ACKNOWLEDGMENTS}

The authors acknowledge technical contributions by Gayle Foster, invaluable assistance from members of the WFUHS Animal Resources Program, and valuable feedback from colleagues in the Department of Microbiology and Immunology. This work was supported by grants from the NIH (AI054425, DC007444, and DC10051).

\section{REFERENCES}

Allen, S., Zaleski, A., Johnston, J. W., Gibson, B. W., and Apicella, M. A. (2005). Novel sialic acid transporter of Haemophilus influenzae. Infect. Immun. 73, 5291-5300.

Anderl, J. N., Franklin, M. J., and Stewart, P. S. (2000). Role of antibiotic penetration limitation in
Klebsiella pneumoniae biofilm resistance to ampicillin and ciprofloxacin. Antimicrob. Agents Chemother. 44, 1818-1824.

Anderl, J. N., Zahller, J., Roe, F., and Stewart, P. S. (2003). Role of nutrient limitation and stationary-phase existence in Klebsiella pneumoniae biofilm resistance to ampicillin and ciprofloxacin. Antimicrob. Agents Chemother. 47, 1251-1256.

Armbruster, C., Byrd, M., Love, C., Juneau, R., Kock, N. D., and Swords, W. E. (2009). LuxS promotes biofilm maturation and persistence of nontypeable Haemophilus influenzae in experimental otitis media by modulation of lipooligosaccharide composition. Infect. Immun. 77, 4081-4091.

Armbruster, C. E., Hong, W., Pang, B., Weimer, K. E., Juneau, R. A., Turner, J., and Swords, W. E. (2010). Indirect pathogenicity of Haemophilus influenzae and Moraxella catarrhalis in polymicrobial otitis media occurs via 
interspecies quorum signaling. MBio 1, 102-110.

Armbruster, C. E., Pang, B., Murrah, K., Juneau, R. A., Perez, A. C., Weimer, K. E., and Swords, W. E. (2011). RbsB (NTHI_0632) mediates quorum signal uptake in nontypeable Haemophilus influenzae strain 86-028NP. Mol. Microbiol. 82, 836-850.

Bakaletz, L. O., Tallan, B. M., Hoepf, T., DeMaria, T. F., Birck, H. G., and Lim, D. J. (1988). Frequency of fimbriation of nontypable Haemophilus influenzae and its ability to adhere to chinchilla and human respiratory epithelium. Infect. Immun. 56, 331-335.

Berenson, C. S., Garlipp, M. A., Grove, L. J., Maloney, J., and Sethi, S. (2006a). Impaired phagocytosis of nontypeable Haemophilus influenzae by human alveolar macrophages in chronic obstructive pulmonary disease. J. Infect. Dis. 194, 1375-1384.

Berenson, C. S., Wrona, C. T., Grove, L. J., Maloney, J., Garlipp, M. A., Wallace, P. K., Stewart, C. C., and Sethi, S. (2006b). Impaired alveolar macrophage response to Haemophilus antigens in chronic obstructive lung disease. Am. J. Respir. Crit. Care Med. 174, 31-40.

Chin, C. L., Manzel, L. J., Lehman, E. E., Humlicek, A. L., Shi, L., Starner, T. D., Denning, G. M., Murphy, T. F., Sethi, S., and Look, D. C. (2005). Haemophilus influenzae from patients with chronic obstructive pulmonary disease exacerbation induce more inflammation than colonizers. Am. J. Respir. Crit. Care Med. 172, 85-91.

Greiner, L., Watanabe, H., Phillips, N. J., Shao, J., Morgan, A., Zaleski, A., Gibson, B. W., and Apicella, M. A. (2004). Nontypeable Haemophilus influenzae strain 2019 produces a biofilm containing $\mathrm{N}$-acetylneuraminic acid that may mimic sialylated O-linked glycans. Infect. Immun. 72, 4249-4260.

Hall-Stoodley, L., Hu, F. Z., Gieseke, A., Nistico, L., Nguyen, D., Hayes, J. D., Forbes, M., Greenberg, D. P., Dice, B., Burrows, A., Wackym, P., Stoodley, P., Post, J. C., Ehrlich, G. D., and Kerschner, J. E. (2006). Direct detection of bacterial biofilms on the middle ear mucosa of children with chronic otitis media. JAMA 296, 202-211.

Hall-Stoodley, L., and Stoodley, P. (2009). Evolving concepts in biofilm infections. Cell. Microbiol. 11, 1034-1043.

Halsey, T. A., Vazquez-Torres, A., Gravdahl, D. J., Fang, F. C., and
Libby, S. J. (2004). The ferritinlike Dps protein is required for Salmonella enterica serovar Typhimurium oxidative stress resistance and virulence. Infect. Immun. 72, 1155-1158.

Harrison, A., Dyer, D. W., Gillaspy, A., Ray, W. C., Mungur, R., Carson, M. B., Zhong, H., Gipson, J., Gipson, M., Johnson, L. S., Lewis, L., Bakaletz, L. O., and Munson, R. S. Jr. (2005). Genomic sequence of an otitis media isolate of nontypeable Haemophilus influenzae: comparative study with $H$. influenzae serotype d, strain KW20. J. Bacteriol. 187, 4627-4636.

Hong, W., Juneau, R., Pang, B., and Swords, W. E. (2009). Survival of bacterial biofilms within neutrophil extracellular traps promotes nontypeable Haemophilus influenzae persistence in the chinchilla model for otitis media. J. Innate Immun. 1 , 215-224.

Hong, W., Mason, K., Jurcisek, J. A., Novotny, L. A., Bakaletz, L. O., and Swords, W. E. (2007a). Phosphorylcholine decreases early inflammation and promotes the establishment of stable biofilm communities of nontypeable Haemophilus influenzae strain 86-028NP in a chinchilla model of otitis media. Infect. Immun. 75, 958-965.

Hong, W., Pang, B., West-Barnette, S., and Swords, W. E. (2007b). Phosphorylcholine expression by nontypeable Haemophilus influenzae correlates with maturation of biofilm communities in vitro and in vivo. J. Bacteriol. 189, 8300-8307.

Jones, P. A., Samuels, N. A., Phillips, N. J., Munson, R. S., Bozue, J. A., Arseneau, J. A., Nichols, W. A., Zaleski, A., Gibson, B. W., and Apicella, M. A. (2002). Haemophilus influenzae type $\mathrm{B}$ strain $\mathrm{A} 2$ has multiple sialyltransferases involved in lipooligosaccharide sialylation. J. Biol. Chem. 277, 14598-14611.

Juneau, R. A., Pang, B., Weimer K. E., Armbruster, C. E., and Swords, W. E. (2011). Nontypeable Haemophilus influenzae initiates formation of neutrophil extracellular traps. Infect. Immun. 79, 431-438.

Jurcisek, J. A., and Bakaletz, L. O. (2007). Biofilms formed by nontypeable Haemophilus influenzae in vivo contain both double-stranded DNA and type IV pilin protein. J. Bacteriol. 189, 3868-3875.

Jurcisek, J. A., Bookwalter, J., Baker, B., Fernandez, S., Novotny, L. A., Munson, R. S. Jr., and Bakaletz,
L. O. (2007). The PilA protein of nontypeable Haemophilus influenzae plays a role in biofilm formation, adherence to epithelial cells and colonization of the mammalian upper respiratory tract. Mol. Microbiol. 65 , 1288-1299.

Jurcisek, J. A., Greiner, L., Watanabe, H., Zaleski, A., Apicella, M. A., and Bakaletz, L. O. (2005). Role of sialic acid and complex carbohydrate biosynthesis in biofilm formation by nontypeable Haemophilus influenzae in the chinchilla middle ear. Infect. Immun. 73 , 3210-3218.

Kim, J., Hahn, J. S., Franklin, M. J., Stewart, P. S., and Yoon, J. (2009). Tolerance of dormant and active cells in Pseudomonas aeruginosa PA01 biofilm to antimicrobial agents. J. Antimicrob. Chemother. 63 , 129-135.

Klein, J. O. (2000). The burden of otitis media. Vaccine 19, S2-S8.

Kweon, S. M., Wang, B., Rixter, D., Lim, J. H., Koga, T., Ishinaga, H., Chen, L. F., Jono, H., Xu, H., and Li, J. D. (2006). Synergistic activation of NF-kappaB by nontypeable $H$. influenzae and S. pneumoniae is mediated by CK2, IKKbetaIkappaBalpha, and p38 MAPK. Biochem. Biophys. Res. Commun. 351, 368-375.

Lewis, K. (2001). Riddle of biofilm resistance. Antimicrob. Agents Chemother. 45, 999-1007.

Moghaddam, S. J., Clement, C. G., De la Garza, M. M., Zou, X., Travis, E. L., Young, H. W., Evans, C. M., Tuvim, M. J., and Dickey, B. F. (2007). Haemophilus influenzae lysate induces aspects of the chronic obstructive pulmonary disease phenotype. Am. J. Respir. Cell Mol. Biol. $38,629-638$.

Murphy, T. F., Brauer, A. L., Grant, B. J., and Sethi, S. (2005a). Moraxella catarrhalis in chronic obstructive pulmonary disease: burden of disease and immune response. Am. J. Respir. Crit. Care Med. 172, 195-199.

Murphy, T. F., Brauer, A. L., Schiffmacher, A. T., and Sethi, S. (2004). Persistent colonization by Haemophilus influenzae in chronic obstructive pulmonary disease. Am. J. Respir. Crit. Care Med. 170 266-272.

Murphy, T. F., and Kirkham, C. (2002). Biofilm formation by nontypeable Haemophilus influenzae: strain variability, outer membrane antigen expression and role of pili. $B M C$ Microbiol. 2, 7.

Murphy, T. F., Kirkham, C., Sethi, S., and Lesse, A. (2005b). Expression of a peroxiredoxin-glutaredoxin by Haemophilus influenzae in biofilms and during human respiratory tract infection. FEMS Immunol. Med. Microbiol. 44, 81-89.

Nair, S., and Finkel, S. E. (2004). Dps protects cells against multiple stresses during stationary phase. J. Bacteriol. 186, 4192-4198.

Pang, B., Hong, W., West-Barnette, S. L., Kock, N. D., and Swords, W. E. (2008a). Diminished ICAM-1 expression and impaired pulmonary clearance of nontypeable Haemophilus influenzae in a mouse model for COPD/emphysema. Infect. Immun. 76, 4959-4967.

Pang, B., Winn, D., Johnson, R., Hong, W., West-Barnette, S., Kock, N., and Swords, W. E. (2008b). Lipooligosaccharides containing phosphorylcholine delay pulmonary clearance of nontypeable Haemophilus influenzae. Infect. Immun. 76, 2037-2043.

Paradise, J. L., Rockette, H. E., Colborn, D. K., Bernard, B. S., Smith, C. G., Kurs-Lasky, M., and Janosky, J. E. (1997). Otitis media in 2253 Pittsburgh-area infants: prevalence and risk factors during the first two years of life. Pediatrics 99, 318-333.

Pichichero, M. E. (2005). Evolving shifts in otitis media pathogens: relevance to a managed care organization. Am. J. Manag. Care 11, S192-S201.

Pichichero, M. E., and Casey, J. R. (2007). Evolving microbiology and molecular epidemiology of acute otitis media in the pneumococcal conjugate vaccine era. Pediatr. Infect. Dis. J. 26, S12-S16.

Post, J. C. (2001). Direct evidence of bacterial biofilms in otitis media. Laryngoscope 111, 2083-2094.

Sanderson, A. R., Leid, J., and Hunsaker, D. (2006). Bacterial biofilms on the sinus mucosa of human subjects with chronic rhinosinusitis. Laryngoscope 116, 1121-1126.

Sethi, S., Evans, N., Grant, B. J., and Murphy, T. F. (2002). New strains of bacteria and exacerbations of chronic obstructive pulmonary disease. N. Engl. J. Med. 347, 465-471.

Sethi, S., and Murphy, T. F. (2001) Bacterial infection in chronic obstructive pulmonary disease in 2000, a state-of-the-art review. Clin Microbiol. Rev. 14, 336-363.

Sethi, S., Sethi, R., Eschberger, K., Lobbins, P., Cai, X., Grant, B. J., and Murphy, T. F. (2007). Airway bacterial concentrations and exacerbations of chronic 
obstructive pulmonary disease. Am. J. Respir. Crit. Care Med. 176, 356-361.

Starner, T. D., Zhang, N., Kim, G., Apicella, M. A., and McCray, P. B. Jr. (2006). Haemophilus influenzae forms biofilms on airway epithelia: implications in cystic fibrosis. Am. J. Respir. Crit. Care Med. 174, 213-220.

Swords, W. E., Moore, M. L., Godzicki, L., Bukofzer, G., Mitten, M. J., and VonCannon, J. (2004). Sialylation of lipooligosaccharides promotes biofilm formation by nontypeable Haemophilus influenzae. Infect. Immun. 72, 106-113.

Tumkaya, M., Atis, S., Ozge, C., Delialioglu, N., Polat, G., and
Kanik, A. (2007). Relationship between airway colonization, inflammation and exacerbation frequency in COPD. Respir. Med. 101, 729-737.

Vattanaviboon, P., and Mongkolsuk, S. (1998). Evaluation of the role hydroxyl radicals and iron play in hydrogen peroxide killing of Xanthomonas campestris pv. phaseoli. FEMS Microbiol. Lett. 169, 255-260.

Watanabe, T., Jono, H., Han, J., Lim, D. J., and Li, J. D. (2004). Synergistic activation of NF-kappaB by nontypeable Haemophilus influenzae and tumor necrosis factor alpha. Proc. Natl. Acad. Sci. U.S.A. 101, 3563-3568.
Wedzicha, J. A., and Seemungal, T. A. (2007). COPD exacerbations: defining their cause and prevention. Lancet 370, 786-796.

West-Barnette, S., Rockel, A., and Swords, W. E. (2006). Biofilm growth increases phosphorylcholine content and decreases potency of nontypeable Haemophilus influenzae endotoxins. Infect. Immun. 74, 1828-1836.

Conflict of Interest Statement: The authors declare that the research was conducted in the absence of any commercial or financial relationships that could be construed as a potential conflict of interest.
Received: 15 December 2011; accepted: 13 April 2012; published online: 03 May 2012.

Citation: Pang B, Hong W, Kock ND and Swords WE (2012) Dps promotes survival of nontypeable Haemophilus influenzae in biofilm communities in vitro and resistance to clearance in vivo. Front. Cell. Inf. Microbio. 2:58. doi: 10.3389/fcimb.2012.00058

Copyright (c) 2012 Pang, Hong, Kock and Swords. This is an open-access article distributed under the terms of the Creative Commons Attribution Non Commercial License, which permits non-commercial use, distribution, and reproduction in other forums, provided the original authors and source are credited. 


\section{APPENDIX}

Upregulated genes of biofilm vs. stationary phase.

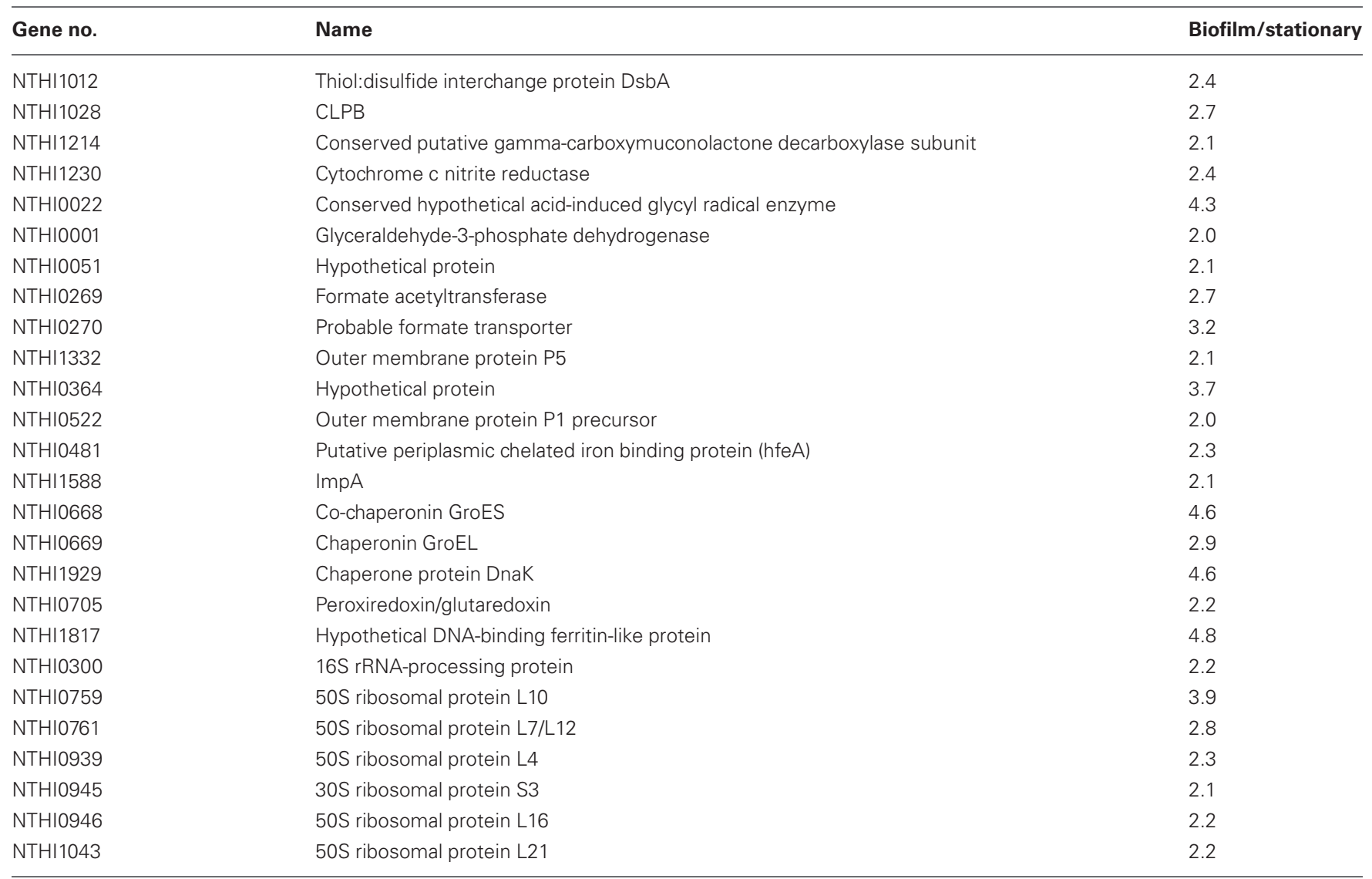

Downregulated genes of biofilm vs. stationary phase.

\begin{tabular}{lll}
\hline Gene no. & Name & Biofilm/stationary \\
\hline NTHI0831 & Tryptophanase & 0.5 \\
NTHI0339 & Hypothetical protein & 0.4 \\
\hline
\end{tabular}

